\title{
Numerical simulation of dust explosions in pneumatic conveyors
}

\author{
U. Bielert, M. Sichel \\ Department of Aerospace Engineering, University of Michigan, Ann Arbor, MI 48109-2118, USA
}

Received: 5 July 1997 / Accepted 21 July 1998

\begin{abstract}
Dust conveyors are used in a wide range of industrial applications. Explosions can be transmitted through dust conveyors to different parts of a processing facility and thus can cause a large amount of damage. In order to study the evolution of dust explosions in such conveyors, a numerical model was developed which combines a front tracking method with a solver for the Euler equations. In this model the effects of the chemical reactions and of the flow turbulence were summarized in the turbulent burning velocity of the dust-air mixture. This approach results in a large reduction of the computational effort and thus allows to study the influence of parameter variations. Here results are presented for corn starch-air mixtures. The numerical model was first calibrated by comparison with one set of experimental data. The model was then tested by comparison with different experimental data and the sensitivity of the model parameters is discussed. Finally calculations were performed for different dust concentrations, flow velocities and tube lengths.
\end{abstract}

Key words: Dust explosions, Turbulent flame propagation, Front tracking, Dust conveyor

\section{Introduction}

A common feature of most dust handling facilities is the need to transport dusts between processing steps. Frequently pneumatic dust conveyors, in which the dust particles are suspended in an air flow, are employed for this task. Such pneumatic dust conveyors have no moving parts in the dust-air mixture, eliminating an important possible ignition source, and can be operated below atmospheric pressure to ensure that no dust leaks from the transport system. Equipment to feed the dust into an air stream and to separate the dust from the air are readily available.

However, from a safety point of view the connections between the different parts of a processing facility pose a major hazard. It is through these connections that an explosion, starting from a flame generated by an ignition source, can be transmitted from one part of the processing facility to another. To make matters worse, the connecting tubes necessarily have a high length to diameter ratio and the interactions between the flow field and a propagating flame in such tubes can produce very large flame velocities and even transition to detonation (Zhang and Grönig 1991). Such rapidly accelerating dust flames in connecting tubes significantly reduce the time available to initiate counter measures such as fast acting valves or explosion

Correspondence to: U. Bielert. Forschungszentrum Karlsruhe, Institut für Neutronenphysik und Reaktortechnik, Postfach 3640, D-76021 Karlsruhe, Germany. E-mail: bielert@inr.fzk.de An abridged version of this paper was presented at the 16th Int. Colloquium on the Dynamics of Explosions and Reactive Systems at Krakow, Poland, from July 27 to August 1, 1997. suppression systems. At the same time such flames significantly increase the overpressure generated during such explosions. The design of efficient explosion protection systems therefore requires some means of predicting the flame propagation and the resulting pressure distributions in the connecting conveyor tubes.

Several experimental studies of dust explosions in tubes have been performed and have been reviewed in (Bartknecht 1987; Eckhoff 1991). In these studies the dustair mixture was either generated by blowing compressed air over a dust layer or by releasing a mixture of compressed air and dust from a reservoir through a set of nozzles into the test apparatus (Zhang and Grönig 1991; Kauffman et al. 1984; Pineau et al. 1980; Pineau and Ronchail 1987). The resulting dust cloud is, in both cases, turbulent but has no mean velocity. These static conditions are different from those in pneumatic dust conveyors which typically operate with mean flow velocities on the order of 10 to $30 \mathrm{~m} / \mathrm{s}$.

Vogl reports experimental results on dust explosions in pneumatic dust conveyors (Vogl 1992, 1995, 1996). These experiments were performed in complete dust conveying systems including a dust feeder and cyclone separator under realistic operating conditions. Reference measurements employing the usual dust dispersion methods showed that the method of dispersion had a significant influence on the resulting flame propagation. In that study Vogl investigated the influence of tube diameter, tube length, initial flow velocity and ignition location on the resulting pressure distribution and on the propagation velocity of the flame front for different dusts over a wide 
range of dust concentrations. One focus of this study was to determine worst case scenarios and limiting cases.

In an experimental investigation, it is generally impossible to study all possible combinations of the relevant parameters. However, the experimental data can be used to calibrate a numerical model for the simulation of the physical processes involved. Such validated models can then be used to predict the effects of parameter combinations not investigated in the experiment (if one remains within the range of the data base). For the assessment of explosion hazards in industrial facilities, numerical models are needed which are capable of predicting critical parameters of explosions, such as the maximum pressure, the rate of pressure rise and the time delay until the flame reaches a given position, with reasonable accuracy. At the same time such models should require only moderate computational effort in order to permit the study of a wide range of parameter variations during the design process. In order to achieve these goals it is necessary to represent some of the complex details of the physical processes involved in the propagation and acceleration of turbulent dust flames by simplified models.

The development of such a model for the simulation of flame propagation in dust conveyer tubes with a mean flow velocity and its validation by comparison to the experimental results of Vogl $(1992,1995,1996)$ is described in the present paper. The model is then applied to the study of dust conveyer explosions for parameters beyond those considered by Vogl. The numerical model is based on a front tracking method (Noh and Woodward 1976; Hirt and Nichols 1981; Bielert 1994). The main feature of this method is the explicit tracking of the leading edge of the turbulent flame zone propagating through the region of interest. In the model considered here the two major effects governing the propagation of the flame front are convective transport of the flame by the flow field and the propagation of the flame into the unburned mixture.

Hence, to apply the front tracking method the mean flow field and the propagation velocity of the flame front $s_{b}$ must be known. Empirical relations are used to represent this velocity and the optimum values of the empirical constants involved are determined by comparison with the experimental results. The flow fields on the two sides of the flame front are calculated from the Euler equations, which are solved by a Godunov method. The model assumes that the dust-oxidizer reactions all occur within the flame front; thus, the need to consider the detailed reaction kinetics and the stiff partial differential equations involved is avoided. The composition of the products of combustion behind the flame is assumed to remain fixed so that it only needs to be calculated once for each dust-air mixture considered.

In the experimental studies of dust conveyor explosions two extreme cases were identified. The highest pressures and fastest flame propagation velocities were observed for ignition near the center of the tube used to represent the conveyor; whereas, ignition near the tube entrance results in relatively low over pressures and flame velocities. The influence of the model parameters used to describe the

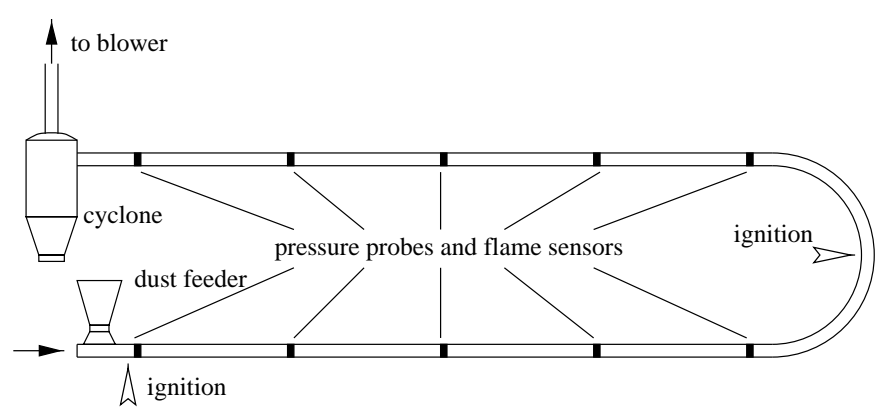

Fig. 1. Experimental setup of Vogl (1995)

propagation of the turbulent flame front on the resulting numerical simulations was investigated for both ignition locations. Using the experimentally validated numerical model additional simulations were then performed for corn starch-air mixtures to determine the effects of dust concentration, initial flow velocity and tube length on dust conveyor explosions.

A brief description of the experiments of Vogl (1992, 1995, 1996) is first presented below. The development and validation of the numerical model is described in detail. The results of the application of the validated model to a range of conveyor operating conditions are then presented.

\section{Experimental investigation}

Experimental studies of the propagation of dust flames in pneumatic conveyors were conducted by Vogl (1992, $1995,1996)$ under realistic operating conditions. The experimental setup is shown schematically in Fig. 1. Air is drawn through a tube ranging in length from 40 to $48 \mathrm{~m}$ by a variable flow rate suction blower. Near the tube entrance dust is fed into the air stream by a dust feeder which permits control of the dust concentration in the tube. Dust and air are separated by a cyclone at the end of the tube just before the air reaches the blower inlet. Access holes in the tube spaced at $4 \mathrm{~m}$ intervals were equipped with pressure transducers and optical flame detectors.

In the experiments steady dust-air flow was established and the actual dust concentration was measured with an optical dust concentration probe. The dust-air mixture was then ignited using pyrotechnic igniters with an ignition energy of 1 or $2 \mathrm{~kJ}$. Two ignition positions were investigated: at the middle of the tube and near the tube entrance.

The experimental conditions used in Vogl's investigation are summarized in Table 1 . However, not all possible combinations of the parameters given in the table were actually investigated.

The pressure and flame arrival data were used to determine average flame velocities and maximum pressures as functions of time and position along the tube. The data published in (Vogl 1992, 1995, 1996) was used to calibrate and validate the numerical model described in the next section. 
Table 1. Experimental conditions investigated by Vogl (1995)

\begin{tabular}{ll}
\hline $\begin{array}{l}\text { dust type } \\
\text { dust concentrations } \mathrm{g} / \mathrm{m}^{3}\end{array}$ & $\begin{array}{l}\text { corn, wheat, lycopodium } \\
75 \text { to } 300\end{array}$ \\
\hline $\begin{array}{l}\text { tube diameter }[\mathrm{mm}] \\
\text { tube length } \mathrm{m}\end{array}$ & $\begin{array}{l}100,150,200 \\
40,48,60\end{array}$ \\
\hline $\begin{array}{l}\text { transport velocity }[\mathrm{m} / \mathrm{s}] \\
\text { ignition location }\end{array}$ & $\begin{array}{l}15,20,25,30 \\
\text { entrance, center }\end{array}$ \\
\hline
\end{tabular}

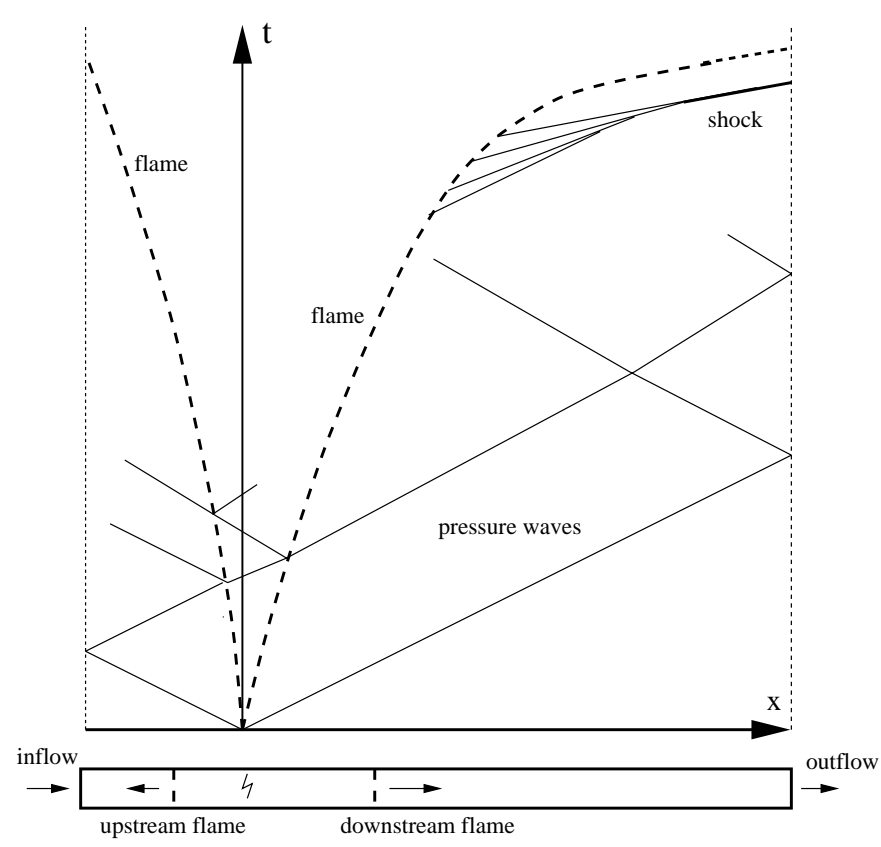

Fig. 2. Schematic representation of numerical simulation

\section{Numerical model}

The system considered in the numerical simulation is shown schematically in Fig. 2. A dust-air mixture flows through a straight tube and is ignited somewhere within the tube. The equipment used in the experimental investigation to produce the dust-air mixture and to separate the dust at the downstream end of the tube are not included in the model. After ignition two flames will propagate through the tube, one upstream against the inflowing dust-air mixture while the second flame propagates downstream in the direction of the flow. Both flames generate pressure waves which, at least initially, propagate faster than the flames. As these pressure waves propagate through the tube and are reflected at the tube ends they interact with other pressure waves and with the flames to produce a complex pattern of partially reflected and partially transmitted waves. If the pressure waves produced by the propagating flame accelerate and steepen to form a single shock a situation can develop in which the flame and the shock travel with the same velocity and become coupled to form a detonation-like structure.

\subsection{Front tracking method}

As indicated in Fig. 2, the propagation of the flames controls the development of the wave structure within the tube. In the numerical model a front tracking method is used to calculate the positions of the flames as a function of time. The front tracking method uses the volume fraction $\mathrm{f}$, a scalar variable, to describe the position of a flame within a cell of the computational grid. The volume fraction $f$ is defined as:

$$
\begin{array}{cl}
f=0 & \begin{array}{l}
\text { the cell contains only reactants } \\
\text { (empty cell) }
\end{array} \\
f=1 & \begin{array}{l}
\text { the cell contains only products } \\
\text { (full cell) }
\end{array} \\
0<f<1 & \begin{array}{l}
\text { the cell contains reactants and } \\
\text { products and thus also an inter- } \\
\text { face. }
\end{array}
\end{array}
$$

From the values of the volume fractions in a local neighborhood the actual position of a flame within a cell can be calculated. Algorithms for the reconstruction of interfaces based on this approach were developed, among others, by Noh and Woodward (1976), Chorin (1989), Hirt and Nichols (1981) and Poo and Ashgriz (1991). A comparison of these four algorithms can be found in Bielert (1994). Here a one-dimensional version of the SLIC algorithm of Noh and Woodward (1976) is used.

The propagation of the flames is controlled by two mechanisms: the convective transport of the interface by the surrounding fluid and the propagation of the interface relative to the fluid, i.e. the actual burning. Both mechanisms are implemented by separate algorithms which determine the volume fractions $f$. This approach assures that changes in topology, which occur when flames merge or fuel pockets burn out, are automatically taken into account.

In order to apply these algorithms the flow field and the propagation velocity of the flame must be known. The calculation of these quantities is discussed in the next sections.

\subsection{Calculation of the flow field}

During combustion a dust-air mixture of reactants with given mass fractions $y_{R_{i}}$ is transformed into a dust-air mixture of products with mass fractions $y_{P_{i}}$. It is assumed that the kinetics of the combustion reactions are confined to the reaction front so that the mass fractions of the combustion products can be assumed to remain constant behind the flame as it propagates through the tube. The product composition thus needs to be calculated only once for each dust-air mixture considered. As already indicated, this approximation eliminates the need to consider detailed reaction kinetics and the time consuming solution of the governing stiff partial differential equations involved. This approach, which has been successfully used in the numerical calculations of gaseous detonation processes (Tonello 1995), of course, neglects any reactions which occur behind the combustion front. 
The composition of the corn starch is taken to be $\left(\mathrm{C}_{6} \mathrm{H}_{10} \mathrm{O}_{5}\right)_{n}$. Then, assuming complete combustion for the part of the corn starch which reacts with the oxygen in the air the governing combustion reaction will be:

$$
\left(\mathrm{C}_{6} \mathrm{H}_{10} \mathrm{O}_{5}\right)_{n}+6 n \mathrm{O}_{2} \longrightarrow 6 n \mathrm{CO}_{2}+5 \mathrm{nH}_{2} \mathrm{O}
$$

It is assumed that all of the corn starch is consumed in lean mixtures, while the portion of corn starch consumed in rich mixtures reacts completely according to the equation above. The composition of the dust-air mixture in a cell containing the flame front and hence reactants and products depends on the volume fraction $\mathrm{f}$ and is given by

$$
y_{i}=(1-f) y_{R_{i}}+f y_{P i}
$$

In order to treat the dust-air mixture as a single phase it is assumed that the particles and air have the same velocity and temperature. This implies that the particles are small and have a high thermal conductivity so that the temperature within the particles is uniform, and that temperature and velocity gradients are moderate. Essentially it is assumed that all non-equilibrium effects are confined to the combustion front which is treated as a discontinuity. As long as the thickness of the actual combustion front is small compared to the characteristic dimension of the problem under consideration this will be a reasonable assumption.

The density of the dust-air mixture is given by

$$
\rho=\frac{m_{g}+m_{s}}{V_{g}+V_{s}} \approx \frac{m_{g}+m_{s}}{V_{g}}=(1+\epsilon) \rho_{g}
$$

with

$$
\epsilon=\frac{m_{s}}{m_{g}}=\frac{y_{s}}{1-y_{s}}
$$

where $y_{s}$ is the mass fraction of the solids in the mixture. The change in $y_{s}$ across the front will depend on the fraction of dust in the reactants which is consumed by the combustion reaction. For a cell containing a mixture of reactants and products $\epsilon$ is given by

$$
\epsilon=f \epsilon_{P}+(1-f) \epsilon_{R}
$$

The internal energy of the dust-air mixture is

$$
e=\rho \sum_{i} y_{i} \int c_{p_{i}} d T
$$

where for the solid species $c_{p_{i}}$ can be replaced by $c_{i}$.

The one-dimensional unsteady Euler equations for the equilibrium dust-air mixture are then

$$
\begin{gathered}
\frac{\partial}{\partial t} \rho+\frac{\partial}{\partial x}(\rho u)=0 \\
\frac{\partial}{\partial t}(\rho u)+\frac{\partial}{\partial x}\left(\rho u^{2}\right)+\frac{\partial p}{\partial x}=0 \\
\frac{\partial e_{t}}{\partial t}+\frac{\partial}{\partial x}\left[\left(e_{t}+p\right) u\right]=\dot{H}
\end{gathered}
$$

with

$$
e_{t}=e+\frac{1}{2} \rho u^{2}
$$

It is assumed that the particles do not contribute to the pressure. Then assuming an ideal gas, the temperature $\mathrm{T}$ of the dust-air mixture is related to the gas density $\rho_{g}$ by

$$
T=\frac{p}{R_{g} \rho_{g}}
$$

The term $\dot{H}$ in the energy equation (10) accounts for heat losses to the surroundings and for additional energy sources as e.g. the ignition energy.

$$
\dot{H}=\dot{H}_{i g n}-\dot{H}_{\text {wall }}
$$

Since the duration of the experimentally observed dust explosions was very short the heat losses to the tube walls were small and were therefore neglected in the simulation so that $\dot{H}_{\text {wall }}=0$.

The ignition energy is an important parameter in the experimental study. In order to approximate the actual ignition process in the simulation, the energy densities in the experiment and the numerical simulation should be similar. To satisfy this condition the rate of energy addition during ignition is calculated from:

$$
\dot{H}_{i g n}=\frac{E_{i g n}}{\Delta t_{i} V_{e x p}}=\frac{E_{i g n}}{\Delta t_{i} d^{2} \frac{\pi}{4} d x}
$$

where $E_{i g n}$ is the experimental value of the ignition energy and $\mathrm{d}$ is the tube diameter which was $200 \mathrm{~mm}$ in the experiments. In the simulation the experimental value of the ignition energy is released during the time interval $\Delta t_{i}$ in one cell of the computational grid.

In the experimental investigation of Vogl (1995) the dust-air mixture was ignited after a homogeneous dust distribution was established in the tube. During the combustion process the suction blower was still working thus influencing the boundary conditions at the downstream end of the tube. While the dust feeder at the tube entrance had only a small influence on the resulting flow field, the cyclone, needed at the tube exit to separate dust and air, certainly changed the flow exiting the tube. In the numerical simulation both boundaries were treated in a simplified form as inflow and outflow boundaries.

During the course of the combustion process there can be inflow or outflow at both ends of the tube. For outflow conditions at both ends the pressure was prescribed as the ambient pressure outside of the tube. The other state variables on the boundary were taken from the state in the last cell of the computational grid.

At the tube entrance the initial state was assumed as the state of the inflowing dust-air mixture. Thus, whenever inflow occurred at the tube entrance, unburned dustair mixture at ambient pressure and temperature entered the tube with the prescribed velocity. This boundary condition was sufficient to simulate the effect of the blower in the experiments.

At the tube exit the state of the dust-air mixture entering the tube in the case of inflow generated by the combustion process is not known. Here it was assumed that 
the dust-air mixture with a composition corresponding to that in the last cell of the computational grid moved with the velocity in the last cell but at ambient pressure and temperature.

The Euler equations (8)-(10) were integrated using a Godunov method on a cell centered grid. In this procedure fluxes across the cell boundaries are calculated at an intermediate time level by solving the Riemann problem at these boundaries. Density is calculated from Eq. (4) and the temperature from Eq. (12) with the composition of the mixture for those cells containing the flame front given by the volume fraction $f$ as indicated above. The thermodynamic properties of the mixture of reactants and products are calculated from the given concentrations using the CHEMKIN package (Kee et al. 1993). As already noted above, the reactant and product compositions only need to be calculated once for each fuel/oxidizer mixture.

An exact Riemann solver is used which employs iterative techniques. The slopes of the dependent variables are limited using the slope limiter of Colella (1985). The subroutines used here are a modified version of software developed by Blumenthal (1996) for shock tube calculations with chemical reactions.

\subsection{Calculation of propagation velocity}

The use of the front tracking method requires a knowledge of the propagation velocity of the front. For the turbulent flames which are tracked in the present model this is the turbulent burning velocity $s_{b}$.

Several parameters influence this velocity. The effects of fuel-oxidizer composition, pressure and temperature can be summarized in the laminar burning velocity $s_{l}$. For gaseous fuels in air these effects have been well documented (see e.g. Metghalchi and Keck 1980; Metghalchi and Keck 1982; Garforth and Rallis 1978; Liu et al. 1989). For dust-air mixtures measurements over a range of dust concentrations and experimental conditions have been made by Krause et al. (1996).

It is well known that the laminar burning velocities observed under quiescent conditions are far exceeded in the presence of turbulence. The effects of turbulence are usually accounted for by the use of correlations of the form $s_{b}=f\left(s_{l}, u^{\prime}\right)$ or $s_{b}=f\left(s_{l}, R e_{u^{\prime}}\right)$.

In calculations for internal combustion engines, however, it was found that the values of the turbulent burning velocity given by the usual steady state correlations are only approached in a finite time since the flame starts as a small laminar kernel and the turbulence needs time to wrinkle the flame and increase the flame surface area (Trautwein 1989; Trautwein et al. 1990; Bielert 1994). This effect was taken into account by introducing an additional exponential expression in the first correlation given above in the form:

$$
\frac{s_{b}}{s_{l}}=1+k_{1}\left(\frac{u^{\prime}}{s_{l}}\right)^{n}\left(1-\exp \left(-k_{2} s_{l} t\right)\right)
$$

The parameter $k_{1}$ essentially controls the magnitude of the influence of turbulent fluctuations on the final value of the turbulent burning velocity. The parameter $k_{2}$ determines how rapidly this final value of the turbulent burning velocity is approached.

In the case of internal combustion engines, for which Eq. (15) was originally developed, turbulence measurements have shown that the turbulence intensity is approximately constant during the combustion process. The turbulence induced during the intake stroke decays after the inlet valves close but this decay is counteracted by the effects of the compression stroke. The situation is somewhat different in dust conveyors in which the dust-air mixture is initially moving at a constant velocity which also implies a constant turbulence level. However, as an explosion is initiated in the conveyor tube the expanding combustion products induce a strong displacement flow which results in a temporal increase in the turbulent intensity. Thus the effect of changes of turbulent intensity during the combustion process had to be included in the empirical expression for the propagation velocity of the turbulent flame front. For steady flow through pipes several investigations have shown that the r.m.s. value of turbulence is proportional to the mean flow velocity (Laufer 1953; Ramaprian and Tu 1983; Tsuji and Morikawa 1982; Tsuji et al. 1984; Tu and Ramaprian 1983). Unfortunately similar experimental data for unsteady pipe flow with chemical reactions is difficult to find. In the present simplified simulation the r.m.s. value of the turbulent velocity fluctuation was therefore assumed to be a constant fraction of the local flow velocity:

$$
u^{\prime}=0.2|\bar{u}|
$$

\subsection{Determination of model parameters}

The equation for the turbulent burning velocity $s_{b}$ adopted here contains two model parameters $k_{1}$ and $k_{2}$ which must be determined from a calibration of the model with experimental data. A simple optimization method is used to select values for these model parameters.

The experimental data used for the calibration included pressure traces at fixed measuring ports, flame arrival times and average flame velocities and the variation of pressures with position along the tube axis. In the numerical simulations, pressure histories are stored at positions corresponding to the measuring ports in the experiment. The simulated pressure and flame front data is processed in the same way as in the experiments in order to produce consistent interpretation. For each experimental data point there is then a corresponding numerical data point. The average of the squares of the differences between the simulated and measured data then provides a measure of the quality of the simulation. Since different data sets are used for the quality function defined below, the data must be non-dimensionalized by appropriate reference values. A quality function $\mathrm{Q}$ characterizing the accuracy of the numerical simulation is then defined as:

$$
Q=\frac{1}{n} \sum_{n}\left(\frac{z_{e n}-z_{s n}}{z_{r n}}\right)^{2}
$$




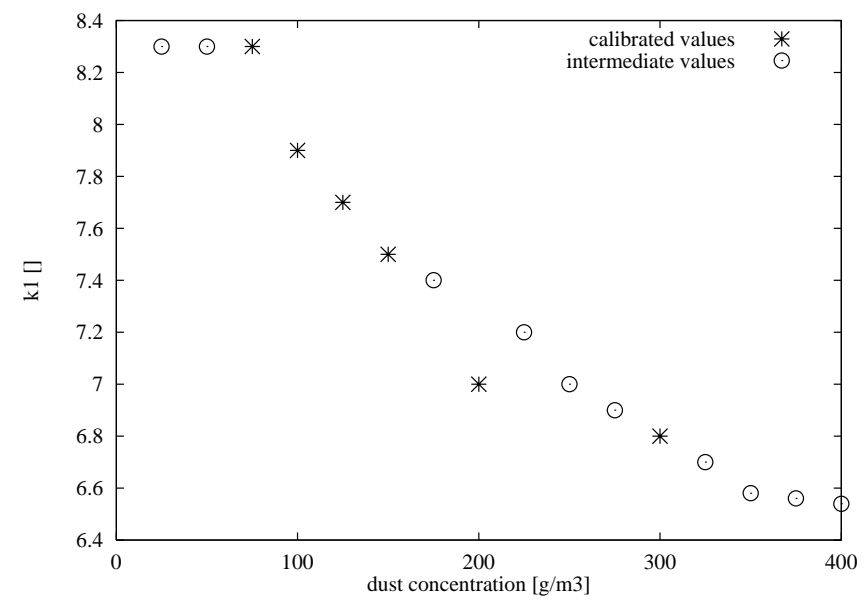

Fig. 3. Model parameter $k_{1}$ as function of dust concentration

Here $z_{e}$ are experimental values, $z_{s}$ are values from the numerical simulation and $z_{r}$ are reference values.

A non-sequential factorial search in the parameter space given by the model parameters $k_{1}$ and $k_{2}$ was then performed to find a local minimum of the quality function $Q$. A set of experiments with the same tube parameters ( $D=200 \mathrm{~mm}, L=40 \mathrm{~m})$ and the same initial flow velocity of $20 \mathrm{~m} / \mathrm{s}$ but with different dust concentrations was selected as reference data for the calibration. The dust-air mixtures in this set were all ignited in the center of the tube. The above optimization procedure was performed for each experiment of the set.

The model parameter $k_{2}$, which characterizes the rate of transition from laminar to turbulent flame propagation, was found to be independent of the dust concentration. However, the model parameter $k_{1}$ which relates the final turbulent burning velocity to the turbulent intensity decreased with increasing dust concentration. This result suggests that the dust had a damping effect on turbulent flame propagation, a result which is, perhaps, not surprising. The values of $k_{1}$ resulting in a minimum in the quality function $\mathrm{Q}$ are shown in Fig. 3 as function of the initial dust concentration. This figure also shows additional values of $k_{1}$ used for dust concentrations for which no calibration with experimental data was performed. The effects of variations in the values of $k_{1}$ and $k_{2}$ on the simulation results are discussed in more detail below.

\section{Results}

\subsection{Comparison with experimental data}

Once the model parameters for the corn starch-air mixtures were determined, numerical simulations were performed for situations for which experimental data were also available. The experimental investigation of Vogl (1995) identified two extreme conditions for dust explosions in long tubes. The most severe explosions with high overpressure and detonation-like flame propagation occur when the dust-air mixture is ignited near the middle of the tube. Only weak explosions with small overpressures are observed when the mixture is ignited near the tube inlet. In this latter situation the combustion products are almost immediately and effectively vented at the end of the tube and the combustion process is then dominated by relatively large flame generated pressure oscillations.

In Fig. 4 pressure histories at six locations along the tube axis are shown for a dust explosion with ignition near the tube entrance. The tube is $40 \mathrm{~m}$ long and the initial flow velocity is $25 \mathrm{~m} / \mathrm{s}$. The dust concentration is $200 \mathrm{~g} / \mathrm{m}^{3}$. The experimental pressure histories are shown as solid lines and the numerical results as dashed lines. At the first five probe positions the general behavior of the local pressure variation is captured reasonably well. Major pressure extrema with the correct amplitude and timing are reproduced by the simulation. However, the oscillations in the numerical simulation are more pronounced; the peaks are sharper and additional oscillations of higher frequencies are observed. The finite diameter of the tube in the experiments allows pressure waves to travel in both axial and radial directions and thus to interfere with one another resulting in a broadening and smoothing of the pressure profiles. In essence, the actual flow is not truly one-dimensional. Pressure waves are diffracted when they are reflected at the end of the tube and viscosity damps the propagating waves. In the one-dimensional model all of these effects are neglected, resulting in more pronounced oscillations. At the last probe position, $2 \mathrm{~m}$ ahead of the tube exit, the amplitude of the pressure oscillations in the experiment decreases compared to that at the other probe positions. This attenuation is attributed to the cyclone separator which is connected to the tube in the experiment and which acts as a large cavity to damp the pressure oscillations. In the idealized boundary conditions used in the numerical simulation the pressure waves are reflected from the tube ends without damping. As a result the agreement between experiment and simulation becomes worse near the tube exit.

Figure 5 compares pressure histories for dust explosions which were ignited near the tube entrance at different flow velocities. The tube is $60 \mathrm{~m}$ long and different dust concentrations were used for the different runs. The experimental data, plotted as solid lines, show increasing amplitudes of oscillation with increasing initial flow velocity. For a given flow velocity the amplitudes are higher at a downstream position of $48 \mathrm{~m}$ than near the ignition location. The numerical simulation captured the general trends, and the simulated pressure histories show similar pressure amplitudes to those from the experiments. However, the details of the pressure histories from the simulation and experiment differed from each other (because the flow was turbulent).

For the cases with end ignition shown in Figs. 4 and 5 the maximum pressures were all below 1.4 bar. When the dust-air mixture is ignited in the middle of the tube the combustion process proceeds more violently with maximum pressures around 20 bar and much faster flame propagation. Typical pressure variations are shown in Fig. 6 for a dust concentration of $200 \mathrm{~g} / \mathrm{m}^{3}$ and an initial flow 

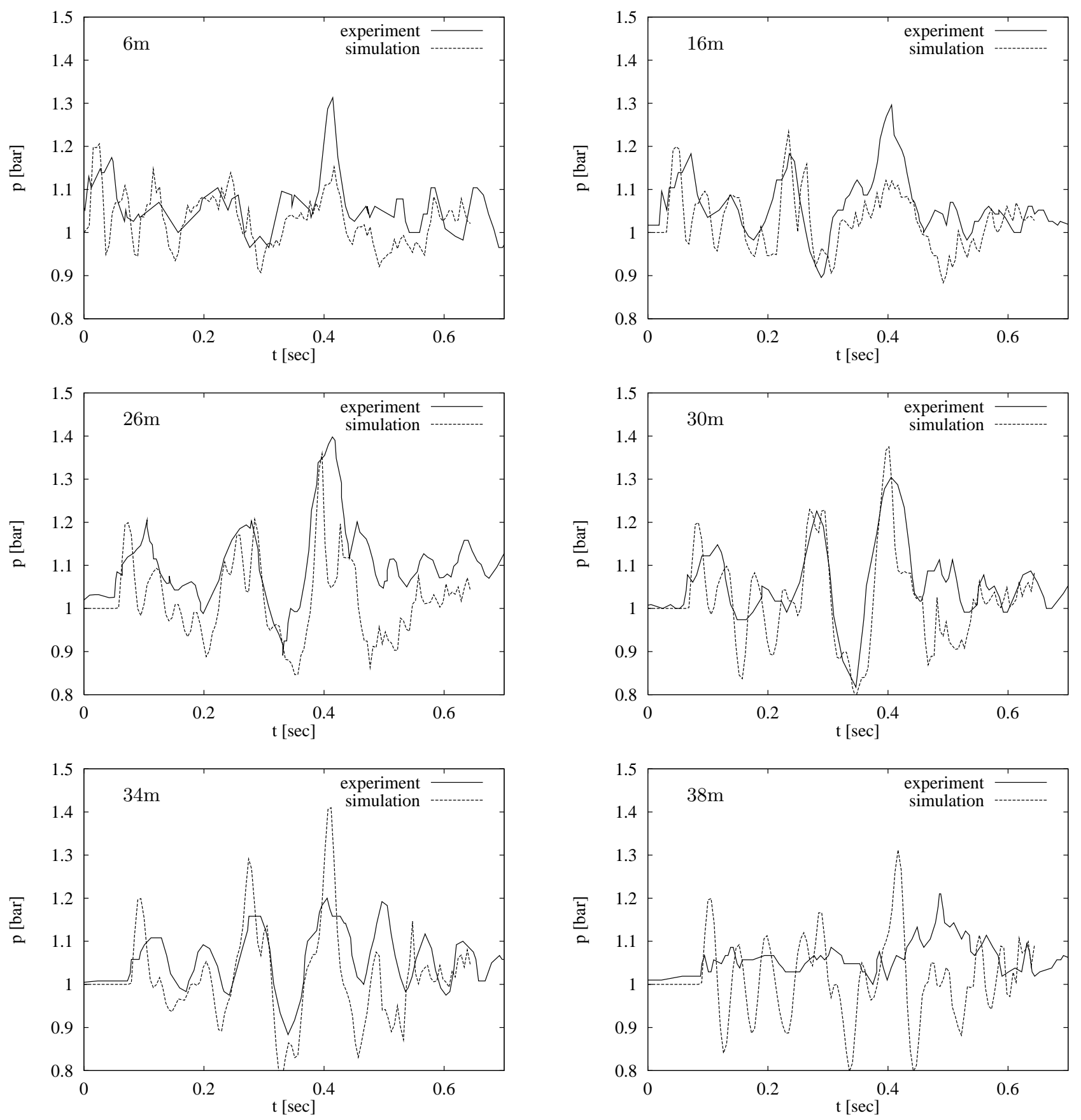

Fig. 4. Comparison of measured and calculated pressure histories at different positions in the tube, DN200, $L=40 \mathrm{~m}, v=$ $25 \mathrm{~m} / \mathrm{s}, c_{d}=200 \mathrm{~g} / \mathrm{m}^{3}$, ignition near tube entrance, experimental data from Vogl (1995)

velocity of $20 \mathrm{~m} / \mathrm{s}$. The corn starch-air mixture was ignited at the center of a $40 \mathrm{~m}$ long tube and pressure histories are given for five positions downstream of the ignition point. Experimental data is again depicted as solid lines and data from the numerical simulation as dashed lines. Close to the ignition location only a small and slow pressure rise is observed in the experiment and the simulation. With increasing distance from the ignition location the rate of pressure rise increases and the maximum pressure reaches higher levels. The numerical simulation shows a pressure maximum which is moving downstream and becoming narrower with higher peak values. The location of this maximum coincides with the pronounced pressure rise observed in the experiment. At the first three locations the experimental pressure continues to increase even after the simulated pressure has reached a maximum value. These differences are probably caused by the influence of the cyclone on the experimental flow field. However, as the pressure peak develops into a shock, and the flame accelerates to supersonic velocities, the influence of the boundary con- 

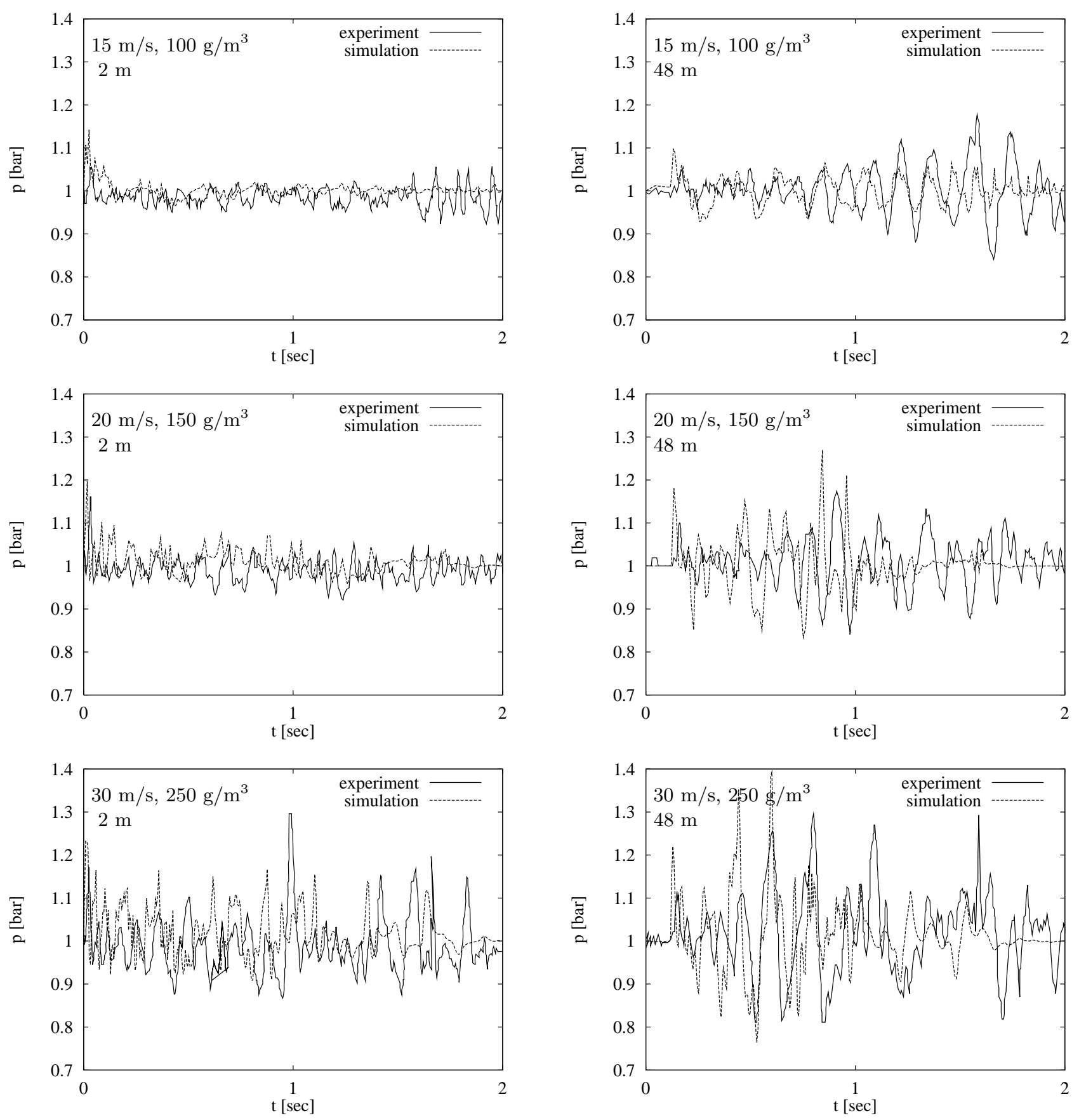

Fig. 5. Comparison of measured and calculated pressure histories for different initial conditions, DN200, $L=60 \mathrm{~m}$, ignition near tube entrance, experimental data from Vogl (1995)

ditions outside of the tube decreases. At the $39 \mathrm{~m}$ position the simulated and measured pressure profiles are in close agreement. Thus it can be assumed that for centered ignition the situation near the tube exit, where the maximum velocities occur, is described with sufficient accuracy by the simplified boundary conditions described above.

Figure 7 shows flame arrival times at different probe positions for a tube of $40 \mathrm{~m}$ length at the top and for a $48 \mathrm{~m}$ long tube at the bottom. Data for different initial flow velocities and different dust concentrations are shown in both diagrams. Experimental data are denoted as symbols, data from the numerical simulation as continuous curves. Early in the combustion process there are some differences between experiment and simulation. For later times closer to the end of the tube the numerical curves lie within the scatter of the experimental data. This agreement is consistent with the agreement between measured and computed pressures near the end of the tube as observed in Fig. 6. The rapidly propagating flames moving downstream are captured more effectively than the slower 

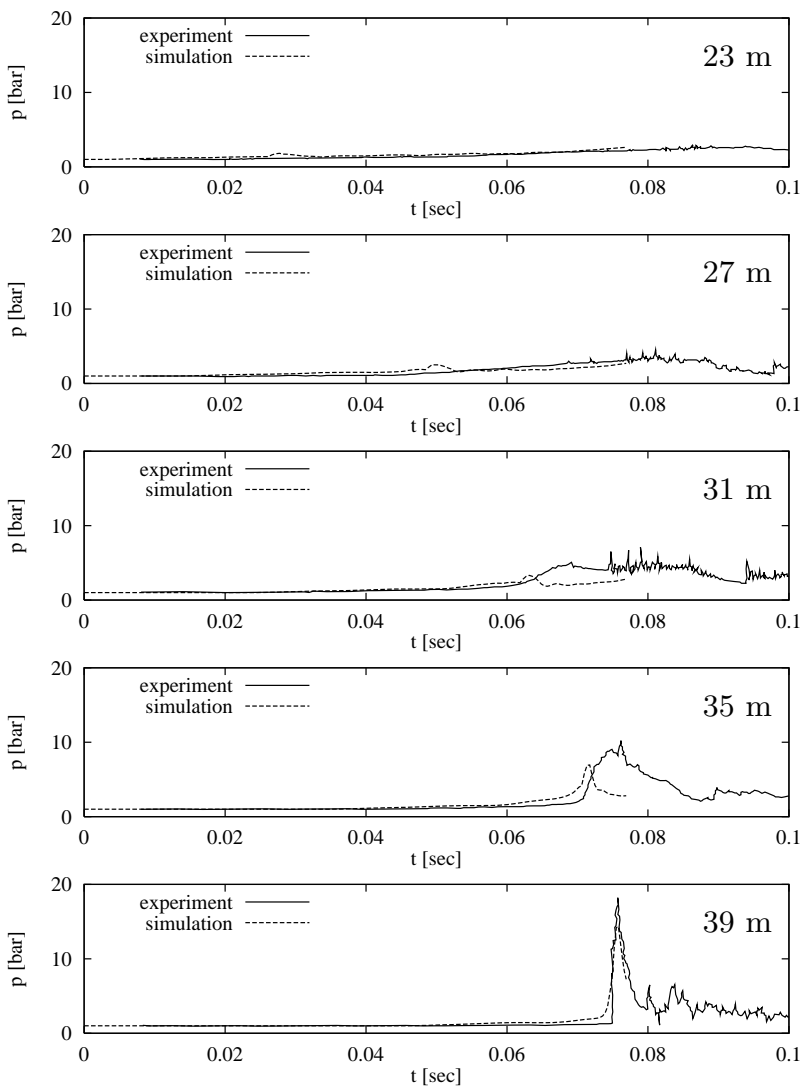

Fig. 6. Comparison of measured and calculated pressure histories, DN200, $L=40 \mathrm{~m}, v=20 \mathrm{~m} / \mathrm{s}, c_{d}=200 \mathrm{~g} / \mathrm{m}^{3}$, ignition at tube center, experimental data from Vogl (1995)

propagating upstream flames. The effect of flow velocity on the flame propagation is very well reproduced by the numerical model.

\subsection{Influence of model parameters $k_{1}$ and $k_{2}$}

The comparison of experimental data with results from numerical simulations indicates that the numerical simulation described above with the appropriate choice of the model parameters $k_{1}$ and $k_{2}$ is capable of reproducing the major effects observed in the experimental investigation of Vogl (1995). For confident use of these parameters, and to obtain a more complete insight into the character of the numerical model it is, however, still desirable to investigate the effects of variations of these parameters on the resulting simulated flame propagation. Results of such parameter variations are shown in Figs. 8 to 11 for a 40 $\mathrm{m}$ long tube with an initial flow velocity of $20 \mathrm{~m} / \mathrm{s}$ and a dust concentration of $200 \mathrm{~g} / \mathrm{m}^{3}$.

The dependence of variation of flame position with time on the parameters $k_{1}$ and $k_{2}$ is shown in Fig. 8 for ignition near the tube entrance. The top plot shows the effect of $k_{1}$ on the flame trajectory for a fixed value of $k_{2}=300 \mathrm{~m}^{-1}$, the value used in the calculations described above. For small values of $k_{1}$ the flame moving upstream is
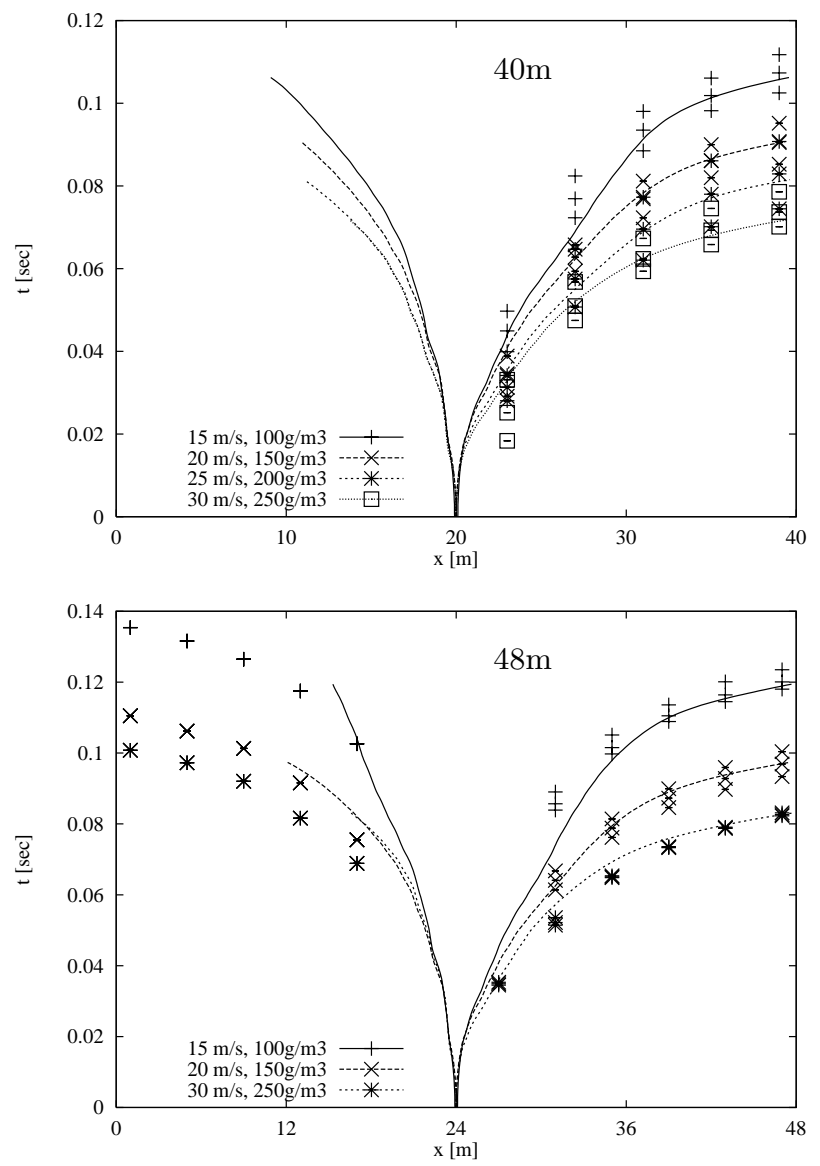

Fig. 7. Comparison of measured and calculated flame positions for different tube lengths, flow velocities and dust concentrations. DN200, ignition at tube center, experimental data from Vogl (1995)

too weak to propagate against the inflowing dust-air mixture and so is convected downstream with the initial flow. With increasing values of $k_{1}$ the flames become faster and stronger so that the upstream propagating flame can exit the tube. For the downstream propagating flame the influence of the parameter $k_{1}$ is much less; however, increasing values of $k_{1}$ result in increasing oscillations in the flame velocity.

The flame accelerates smoothly for $k_{1}<3.0$. Since the flame is then not strong enough to produce strong pressure waves which are reflected at the tube ends and then interact with the flame. For $k_{1} \geq 8.0$ the downstream propagating flame finally develops into a detonation. The pressure waves generated by the flame can steepen into shock waves and the resultant flame-shock complex then propagates through the tube as a detonation-like structure. In the intermediate range of $k_{1}$ values the interactions of the flame with reflected pressure waves become dominant. The flame position at a given time now strongly depends on the complete history of the flame-pressure wave interactions, and the curves for different values of $k_{1}$ intersect frequently. This behavior is almost completely independent of the parameter $k_{2}$ as can be seen in the bottom part of 

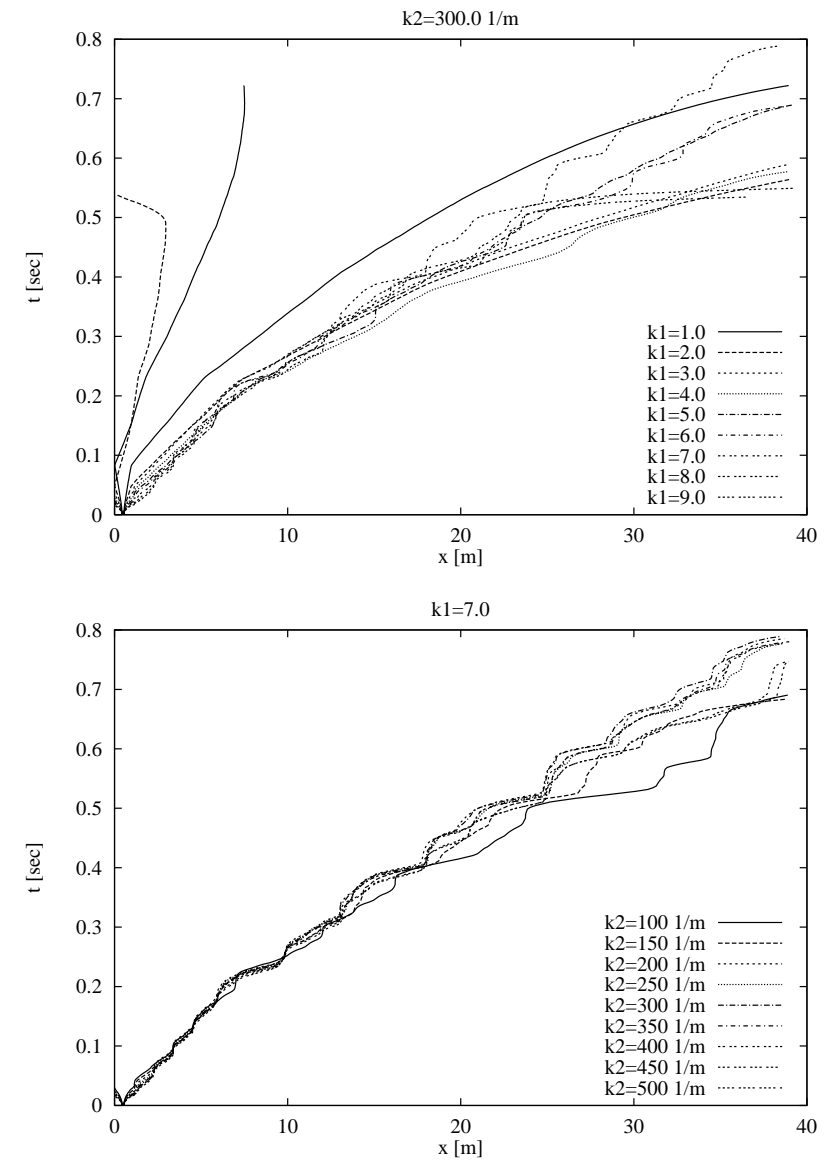

Fig. 8. Position time histories for various combinations of the model parameters $k_{1}$ and $k_{2}$. Top: variation of $k_{1}$ for $k_{2}=$ $300 \mathrm{~m}^{-1}$. Bottom: variation of $k_{2}$ for $k_{1}=7.0$. DN200, $L=$ $40 \mathrm{~m}, v=20 \mathrm{~m} / \mathrm{s}, c_{\mathrm{d}}=200 \mathrm{~g} / \mathrm{m}^{3}$, ignition near tube entrance

Fig. 8. Here the parameter $k_{2}$ was varied for a fixed value of $k_{1}=7.0$. All curves show similar oscillations. As the flame progresses further downstream the curves become more distinct as the differences between their trajectories increase. However, these differences in the flame propagation are local and have only a small influence on the overall process.

Figure 9 shows the maximum pressures and average front velocities which were computed for different combinations of $k_{1}$ and $k_{2}$. The two plots at the top show data for the same parameter combinations as in Fig. 8. The two plots at the bottom show a 3 -D representation of these parameters for a wider range of parameter combinations. The average front velocity is calculated over the whole distance the flame travels from the ignition point to the tube exit. For values of $k_{1}<8$ the pressure increases only slightly with increasing $k_{1}$ and decreases slightly with increasing $k_{2}$. A maximum pressure below 2.0 bar can be expected for this range of parameters. For larger values of $k_{1}$ pressures of the order of 14 bar were reached. The average front velocity is, as already indicated by Fig. 8, not significantly influenced by the parameter $k_{2}$. The values of the average front velocity for different values of $k_{1}$ do show

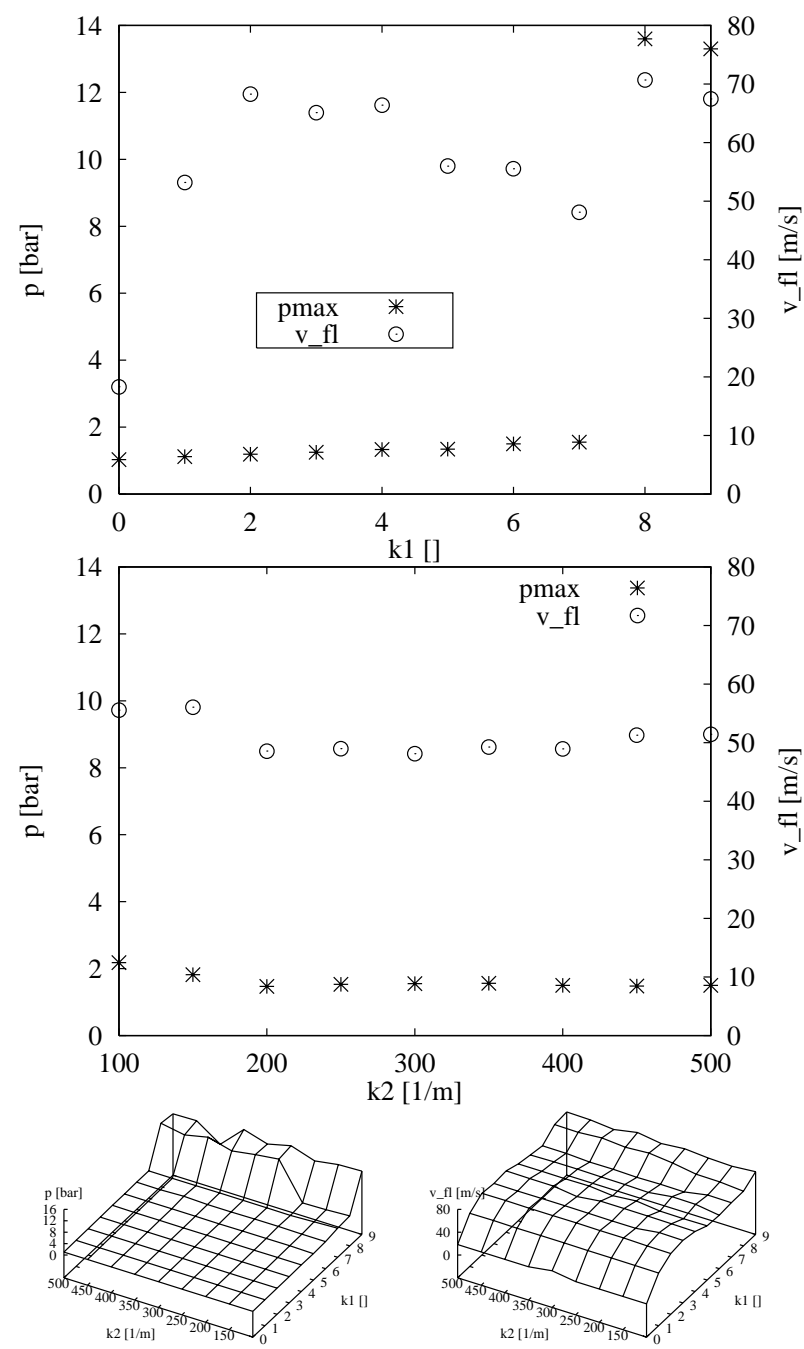

Fig. 9. Maximum pressure and average front velocity as function of the model parameters $k_{1}$ and $k_{2}$. DN200, $L=40 \mathrm{~m}$, $v=20 \mathrm{~m} / \mathrm{s}, c_{\mathrm{d}}=200 \mathrm{~g} / \mathrm{m}^{3}$, ignition near tube entrance

some scatter and seem to decrease slightly with increasing $k_{1}$. However, for values of $k_{1}>8$ the average front velocities increase indicating a change in the character of the solution.

The effects of parameter variations discussed so far were calculated for ignition near the tube entrance. Similar calculations were performed for the same parameter combinations and initial conditions, but for ignition at the tube center. These results are shown in Figs. 10 and 11. The flame position data shown in Fig. 10 indicates a more pronounced dependence on both model parameters $k_{1}$ and $k_{2}$. With increasing values of $k_{1}$ both flames propagate faster relative to the moving fresh mixture and reach the ends of the tube earlier. The final slopes of the flame position curves decrease with increasing $k_{1}$ indicating an increase in the average front velocities. The flame position curves for different values of $k_{2}$ with fixed $k_{1}$ show that the flame accelerates to the same final velocity more rapidly with increasing $k_{2}$. 

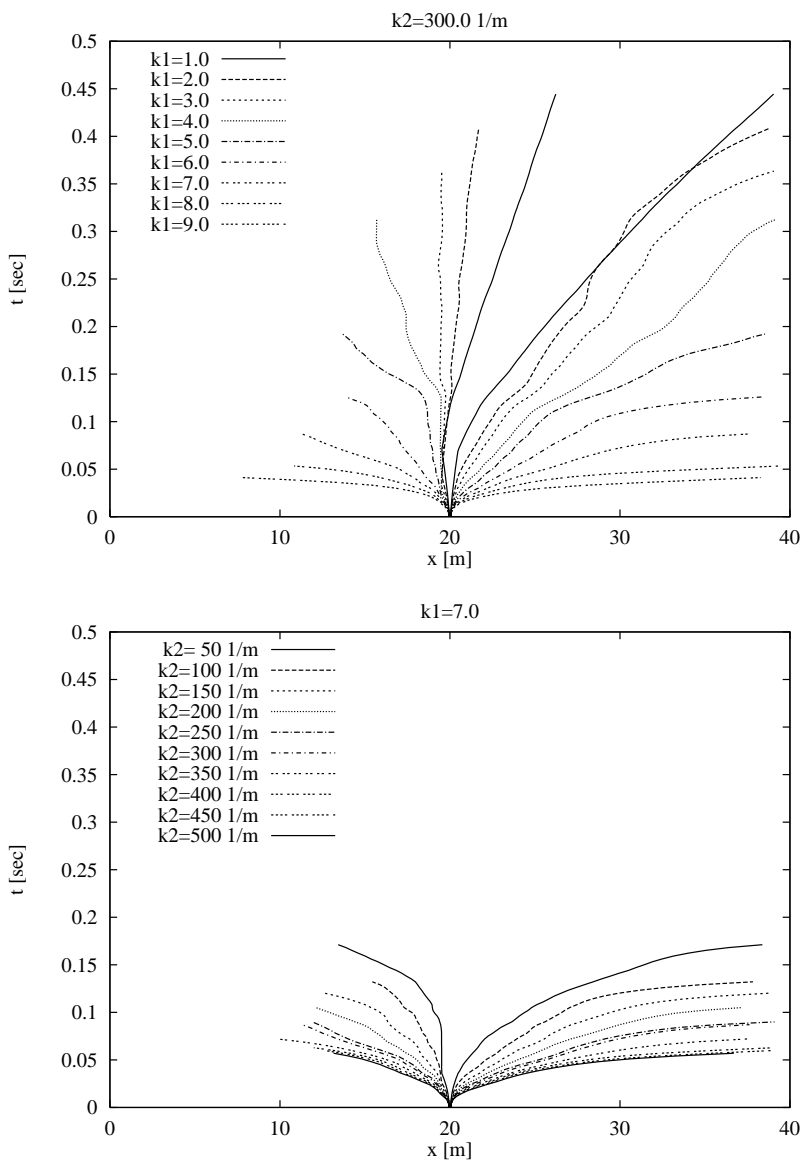

Fig. 10. Position time histories for various combinations of the model parameters $k_{1}$ and $k_{2}$. Top: variation of $k_{1}$ for $k_{2}=$ $300 \mathrm{~m}^{-1}$. Bottom: variation of $k_{2}$ for $k_{1}=7.0$. DN200, $L=$ $40 \mathrm{~m}, v=20 \mathrm{~m} / \mathrm{s}, c_{\mathrm{d}}=200 \mathrm{~g} / \mathrm{m}^{3}$, ignition at tube center

The flame trajectories are relatively smooth indicating that flame pressure wave interactions play a less import role with central ignition. This result is not surprising since central ignition results in higher flame propagation velocities and generates higher pressures than for end ignition. Consequently the relative effect of flame generated pressure perturbations is significantly less.

The maximum pressure and the average front velocity for these calculations are shown in Fig. 11. Both $p_{\max }$ and $v_{f l}$ now increase with increasing $k_{1}$ and $k_{2}$. It should be noted that the front velocities are now much higher than in the end ignition case.

\subsection{Influence of dust concentration, flow velocity and tube length}

The validated numerical model described above now makes it possible to study dust conveyor explosions for values of the governing parameters beyond those considered in the experiments used for validation. The above model is now used to conduct "numerical experiments" to study the effects of dust concentration, initial conveyor flow velocity, and conveyor length on such explosions.

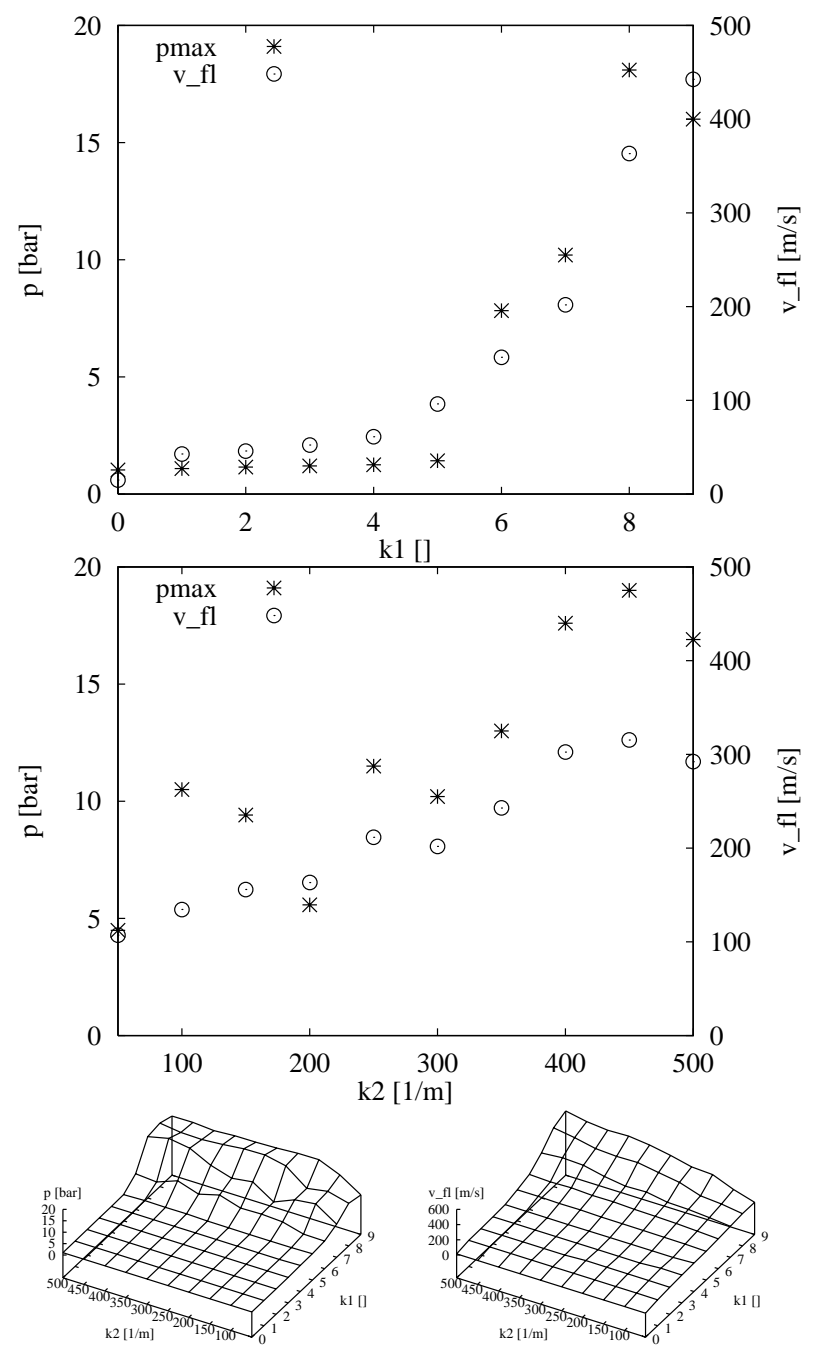

Fig. 11. Maximum pressure and average front velocity as function of the model parameters $k_{1}$ and $k_{2}$. DN200, $L=40 \mathrm{~m}$, $v=20 \mathrm{~m} / \mathrm{s}, c_{\mathrm{d}}=200 \mathrm{~g} / \mathrm{m}^{3}$, ignition at tube center

The influence of dust concentration on the flame propagation in a $40 \mathrm{~m}$ long tube for an initial velocity of 20 $\mathrm{m} / \mathrm{s}$ was investigated for both ignition locations. Calculations were performed for dust concentrations ranging from $25 \mathrm{~g} / \mathrm{m}^{3}$ to $400 \mathrm{~g} / \mathrm{m}^{3}$. Figure 12 shows the flame position data, the maximum pressures and the average front velocities calculated for ignition near the tube entrance. The highest flame speed was observed for the leanest mixture with a dust concentration of $25 \mathrm{~g} / \mathrm{m}^{3}$. Between $25 \mathrm{~g} / \mathrm{m}^{3}$ and $125 \mathrm{~g} / \mathrm{m}^{3}$ the average front velocity decreases and the combustion duration consequently increases. The flame position data for all dust concentrations above $75 \mathrm{~g} / \mathrm{m}^{3}$ fall into one relatively narrow band where the curves for different dust concentrations frequently intersect. The unsteady propagation of the front indicates the importance of flame pressure wave interactions for all but the leanest mixtures. The average front velocities, shown in the lower half of Fig. 12, are almost constant near the stoichiometric concentration of $200 \mathrm{~g} / \mathrm{m}^{3}$ and have a local maximum 

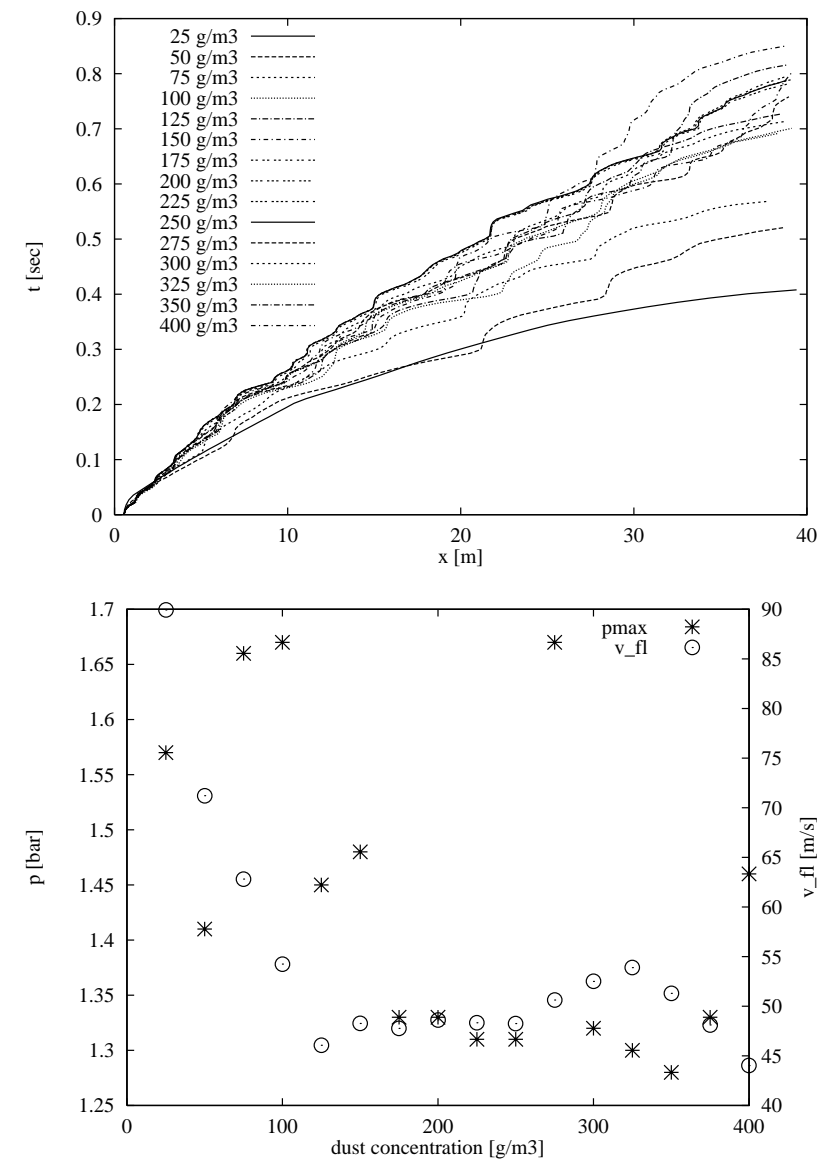

Fig. 12. Variation of dust concentration. DN200, $L=40 \mathrm{~m}$, $v=20 \mathrm{~m} / \mathrm{s}$, ignition near tube entrance

near a concentration of $325 \mathrm{~g} / \mathrm{m}^{3}$. The maximum pressure shows a large scatter with values between 1.3 and 1.7 bar. Mixtures close to stoichiometric do not result in more violent flame propagation, suggesting that maximum energy release seems not to be the controlling parameter. This behavior may be related to the increased density of the dust suspension for the richer mixtures.

For ignition at the center of the tube the results were different. Here, because of confinement, the energy released by the chemical reactions is fully used to accelerate the combustion process. A higher energy release thus results in a more severe explosion. Results for centered ignition are shown in Fig. 13. The flame trajectories are now distinct, and the flame speed increases as the dust concentration approaches stoichiometric conditions. A maximum pressure of almost 16 bar was calculated for the stoichiometric mixture $\left(200 \mathrm{~g} / \mathrm{m}^{3}\right)$. The maximum pressure for the leanest mixture $\left(25 \mathrm{~g} / \mathrm{m}^{3}\right)$ is only little above $1.0 \mathrm{bar}$, suggesting the approach to the lean dust explosion limit. For the rich mixtures the maximum pressure, while below the stoichiometric value, still remains at a significant level. This behavior can be explained by the well-known fact that dust-air mixtures have extremely high rich explosion limits since the fuel occupies only a very small volume in these mixtures.
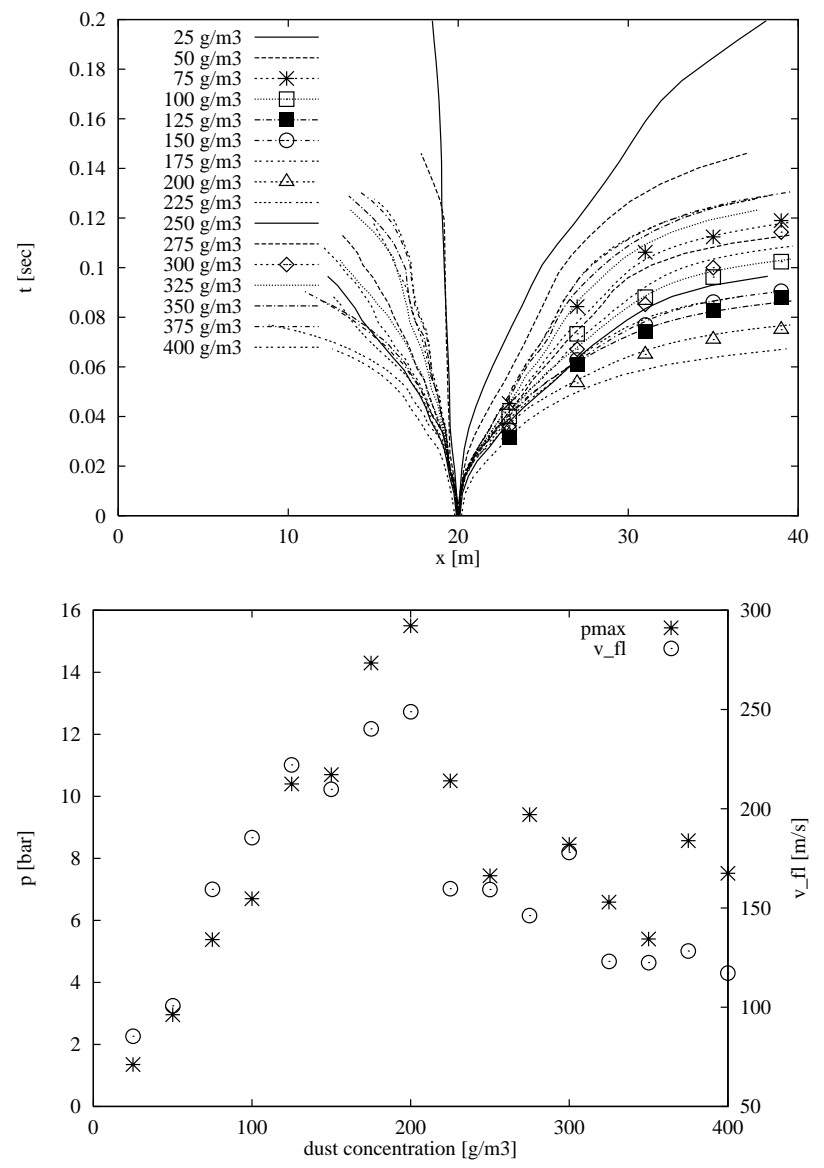

Fig. 13. Variation of dust concentration. DN200, $L=40 \mathrm{~m}$, $v=20 \mathrm{~m} / \mathrm{s}$, ignition at tube center, experimental data from Vogl (1995)

In Fig. 13 experimental data from Vogl (1995) has been included as symbols in the flame position plots. The calculated flame trajectories are in excellent agreement with the available experimental data for the downstream propagating flames.

Variation of the initial flow velocity should also have a large influence on the flame propagation for both ignition locations as has already been observed in Figs. 5 and 7 . A systematic study of the effects of the variation of the initial flow velocity was performed for a tube length of $40 \mathrm{~m}$ and a dust concentration of $200 \mathrm{~g} / \mathrm{m}^{3}$. For ignition at the tube entrance the results are shown in Fig. 14. With increasing initial flow velocity the propagation velocity of the flame can be seen to increase. The flames accelerate only moderately and show some interactions with pressure waves. For velocities below $30 \mathrm{~m} / \mathrm{s}$ the maximum pressures remain below 2.0 bar and the average front velocity increases almost linearly with the initial flow velocity. For higher initial flow velocities maximum pressures increase to above 14 bar and the variation of the front velocity with the initial flow velocity is no longer linear. The results of the corresponding calculations for central ignition are plotted in Fig. 15. For a given flow velocity, the maximum acceleration of the flames is now much larger. Especially for the case of a mixture which is quiescent prior 

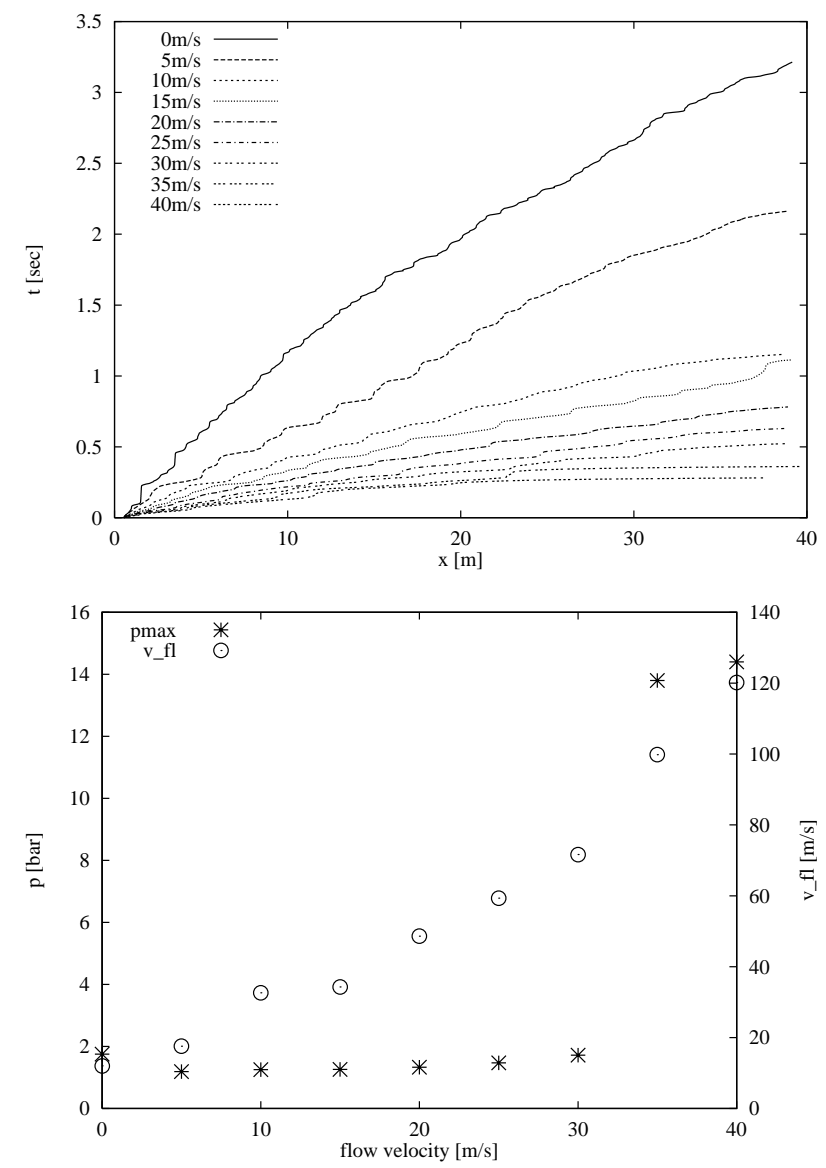

Fig. 14. Variation of flow velocity. DN200, $L=40 \mathrm{~m}, c_{\mathrm{d}}=$ $200 \mathrm{~g} / \mathrm{m}^{3}$, ignition near tube entrance

to ignition the flame initially propagates very slowly and a relatively large fraction of the total combustion time elapses before the flames finally accelerate. With increasing flow velocity there is an increasing difference between the corresponding upstream and downstream flames. The maximum pressure and the average front velocity increase almost linearly with the initial flow velocity over the entire parameter range.

Finally calculations were performed for different tube lengths ranging from 10 to $100 \mathrm{~m}$ for a flow velocity of 20 $\mathrm{m} / \mathrm{s}$ and a dust concentration of $200 \mathrm{~g} / \mathrm{m}^{3}$. Results for ignition near the tube entrance are shown in Fig. 16. The flames propagate slightly faster in shorter tubes as can be seen from the flame trajectories. However, the variation of the average front velocities with the tube length does show some scatter. The tube length is an important parameter for the feedback mechanism between the flames and the pressure waves so that changes in the tube length should result in some variations. The maximum pressure, which remains below 1.5 bar, increases with the tube length. For central ignition, for which results are shown in Fig. 17, there is insufficient time in the shorter tubes for the formation of a detonation-like structure. The maximum pressure increases significantly with tube length (for tubes shorter than $30 \mathrm{~m}$ ). As the tube lengths increase from 30 to $50 \mathrm{~m}$,
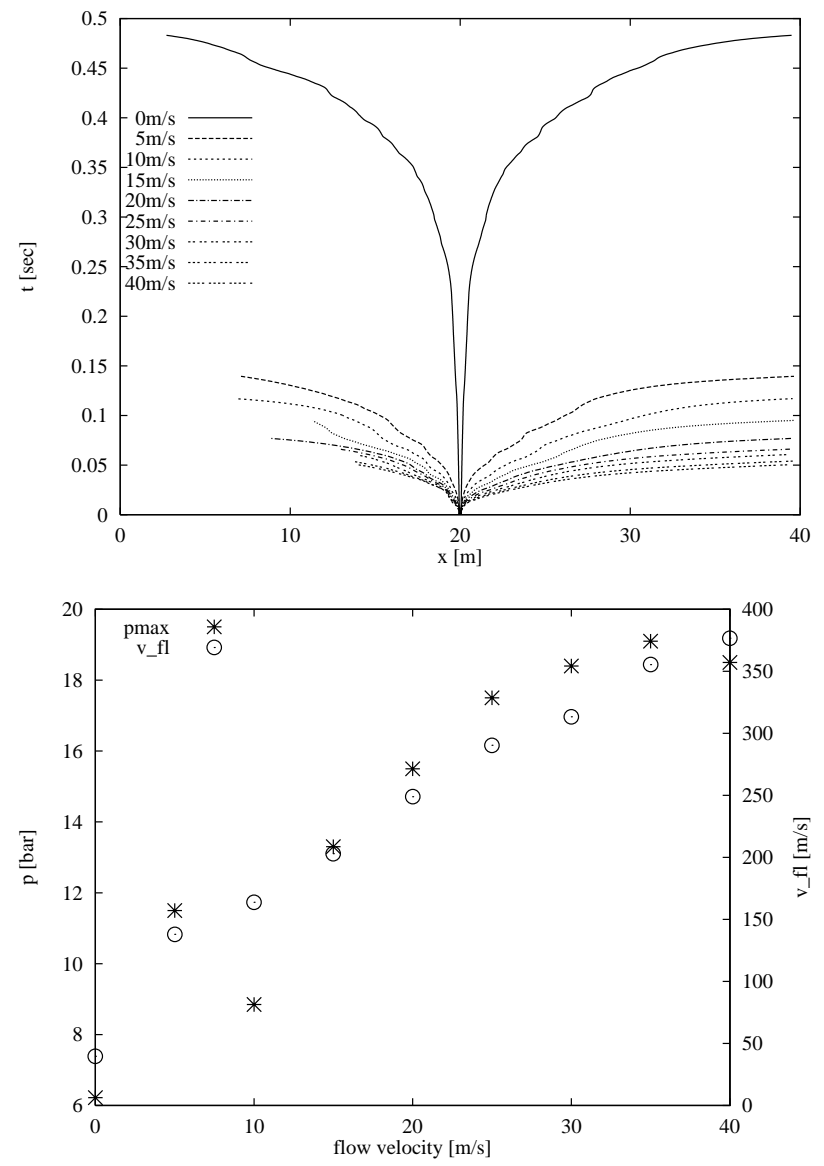

Fig. 15. Variation of flow velocity. DN200, $L=40 \mathrm{~m}, c_{\mathrm{d}}=$ $200 \mathrm{~g} / \mathrm{m}^{3}$, ignition at tube center

a high velocity flame is established resulting in a large increase in the maximum pressure to about 20 bar. Beyond $50 \mathrm{~m}$ the pressure remains approximately constant at about 22 bar. The flame trajectories, which are also shown in this figure, provide an explanation for this behavior. For the shorter tubes there is never enough time for a significant increase in flame velocity. However, as soon as the tube exceeds a critical length a transition occurs to a detonation-like front moving with a constant speed. The average front velocity is calculated over the entire flame path; consequently, the average velocity increases linearly with the tube length.

\section{Discussion and conclusions}

A numerical model employing a front tracking method was applied to the study of turbulent flame propagation in dust conveyors. The model explicitly tracks the leading edge of the turbulent reaction zone. The front tracking method takes into account the convective transport of the flame due the flow of the dust-air mixture and the propagation of the flame with the turbulent burning velocity relative to the reactants. The flow field is calculated using 

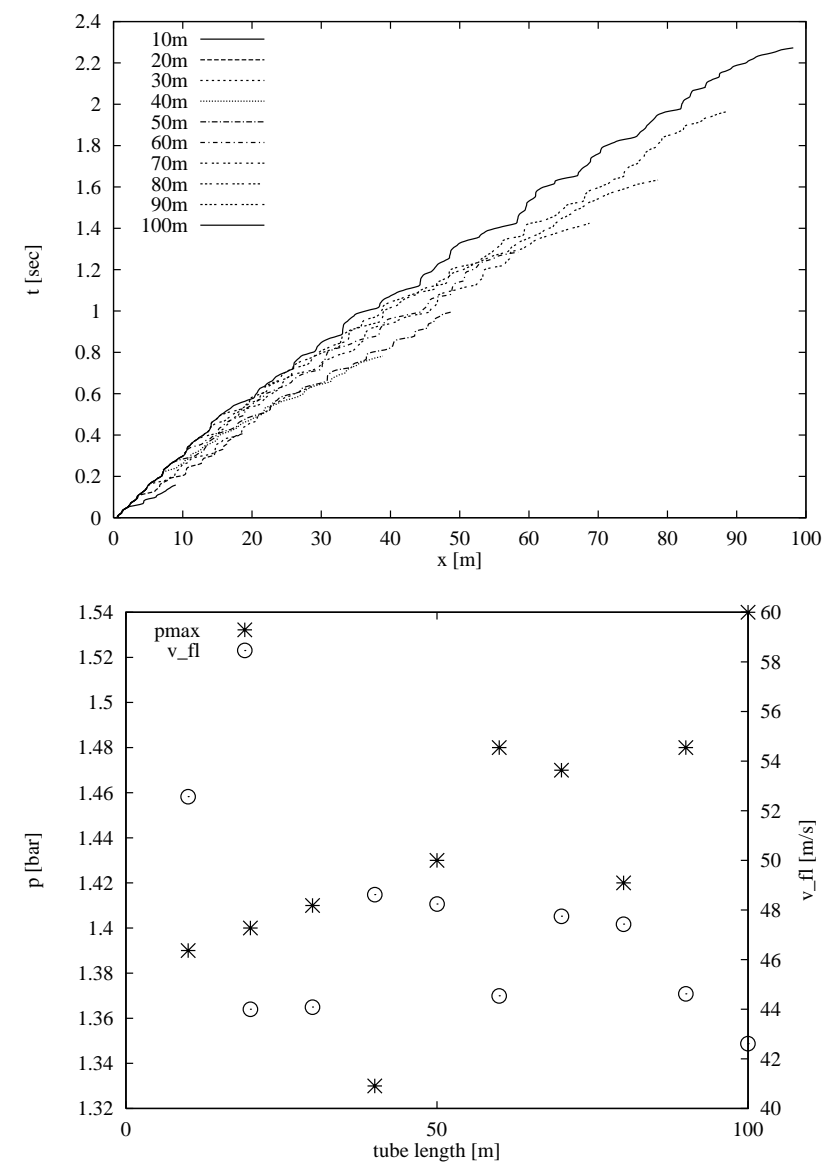

Fig. 16. Variation of tube length. DN200, $v=20 \mathrm{~m} / \mathrm{s}, c_{\mathrm{d}}=$ $200 \mathrm{~g} / \mathrm{m}^{3}$, ignition near tube entrance

a Godunov method to solve the Euler equations. For the dust-air mixture equilibrium between the gas and particle phases was assumed so that the dust-air mixture could be treated as a single phase. The turbulent burning velocity was represented by a global model which takes into account the influence of the fuel/air mixture, the turbulence intensity and time.

A simple optimization technique was used to determine two free parameters of the model by comparing numerical results to experimental data for corn starch-air mixtures. It was found that the parameter $k_{2}$ could be assumed constant for corn starch-air mixtures. The parameter $k_{1}$ was found to be a function of dust concentration.

Additional calculations with these fixed model parameters were then performed and the numerical results were compared to experimental data. These calculations verified that the numerical model captured the major effects of turbulent flames propagation in dust-air mixtures under the conditions corresponding to those in dust conveyors. However, due to factors neglected in the simplified approach of the one-dimensional model, not all details of the experimental results could be reproduced.

The two different explosion processes which were observed in experimental investigations (Vogl 1992, 1995, 1996), namely the development of a detonation-like reaction front in the case of ignition at the tube center and
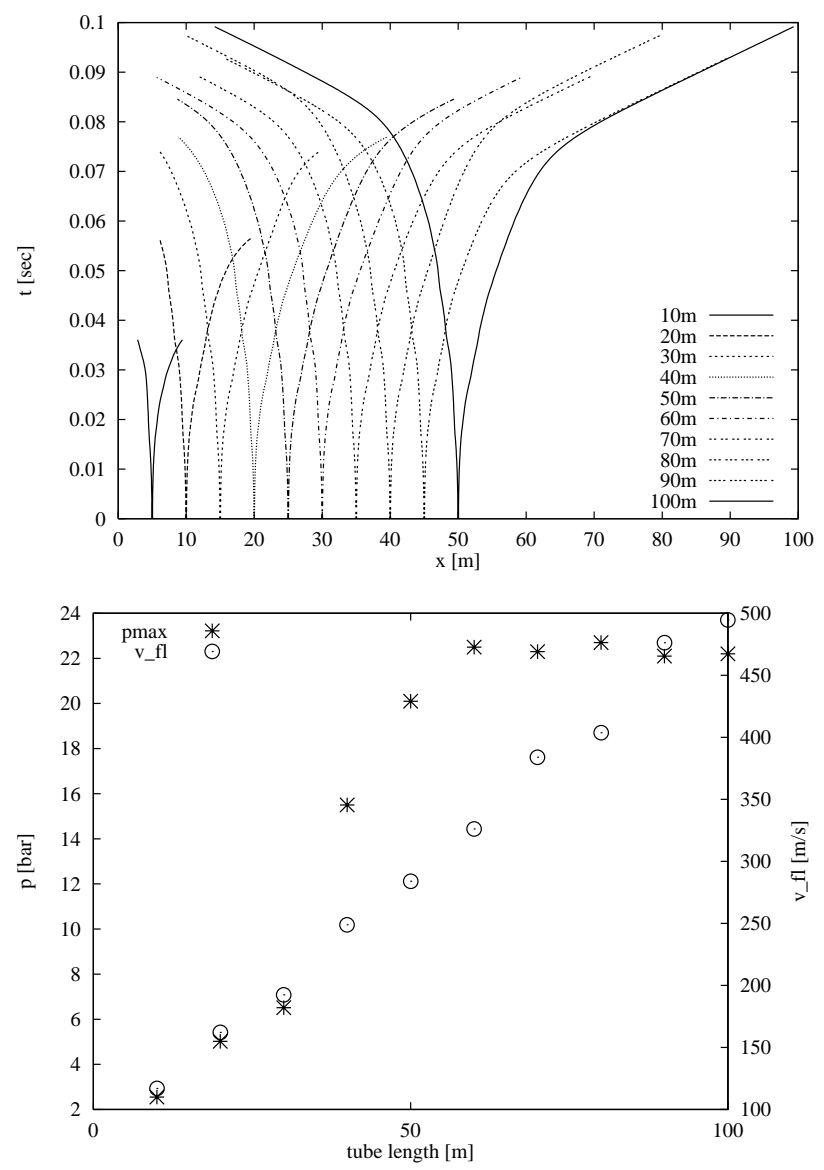

Fig. 17. Variation of tube length. DN200, $v=20 \mathrm{~m} / \mathrm{s}, c_{\mathrm{d}}=$ $200 \mathrm{~g} / \mathrm{m}^{3}$, ignition at tube center

the explosion with a slower moving oscillating front in the case of ignition near the tube entrance, could both be reproduced with the same set of model parameters.

The effects of the model parameters on the results of the numerical simulations were discussed. It could be verified that the numerical results depend in a predictable way on the model parameters.

The numerical model was then used to calculate the effects of variations of dust concentration, initial flow velocity and tube length for corn starch-air mixtures for both tube entrance and centered ignition conditions. The results of these calculations, which required only a modest computational effort, were consistent and provided an example of how a relatively simple but validated numerical scheme can be used to estimate burning and explosion parameters in dust conveyors over a wide range of operating conditions.

Here only calculations with corn starch-air mixtures were performed. But the approach can easily be applied to other dust-air mixtures, particularly since the need to consider detailed reaction kinetics is avoided by assuming that all kinetic processes occur only across the flame front. In the present simulations the influence of the tube diameter and wall boundary layer on the explosion process was not considered. 
The front tracking method as well as the Godunov method have successfully been tested in two-dimensional applications. Therefore it seems promising to also extend the present combination of the two methods to the solution of two-dimensional problems. Such a model would then allow simulations of flames propagating through equipment with complex geometries. Configurations consisting of different vessels and connecting tubes could be studied efficiently with such a numerical tool.

\section{References}

Ashgriz N, Poo JY (1991) FLAIR: Flux Line-Segment Model for Advection and Interface Reconstruction. Journal of Computational Physics, 93:449-468

Bartknecht W (1987) Staubexplosionen - Ablauf und Schutzmassnahmen. Springer Verlag, Heidelberg

Bielert U (1994) Numerische Simulation turbulenter Verbrennungsvorgänge unter motorischen Bedingungen mit einem Front Tracking Verfahren. Fortschritt-Berichte VDI, Reihe 12, Nr. 223. VDI Verlag, Düsseldorf. Dissertation, RWTH Aachen

Blumenthal R (1996) Experimentelle Untersuchung und numerische Simulation der Selbstzündung von Kraftstoff/Luft-Gemischen im Stoßwellenrohr unter Berücksichtigung strömungsmechanischer Einflüsse. Dissertation, RWTH Aachen

Chorin AJ (1989) Flame Advection and Propagation Algorithms. Journal of Computational Physics, 35:1-11

Colella P (1985) Siam J. SCI. STAT. COMPUT., 6(1):104

Eckhoff RK (1991) Dust Explosions in the Process Industries. Butterworth-Heinemann, 1st edition

Garforth AM, Rallis CJ (1978) Laminar Burning Velocity of Stoichiometric Methane-Air: Pressure and Temperature Dependence. Combustion and Flame, 31:53-68

Hirt CW, Nichols BD (1981) Volume of Fluid (VOF) Method for the Dynamics of Free Boundaries. Journal of Computational Physics, 39:201-225

Krause U, Kasch T, Gebauer B (1996) Velocity and concentration effects on the laminar burning velocity of dust-air mixtures. International Symposium on Hazards, Prevention and Mitigation of Industrial Explosions, The Seventh International Colloquium on Dust Explosions, Christian Michelsen Research AS, Bergen, Norway

Kee RJ, Rupley FM, Miller JA (1993) CHEMKIN-II: a FORTRAN Chemical Kinetics Package for the Analysis of Gas Phase Chemical Kinetics. Sandia Report SAND89-8009B UC-706, Sandia National Laboratories

Kauffman CW, Srinath SR, Tezok FI, Nicholls JA, Sichel M (1984) Turbulent and Accelerating Dust Flames. In 20th Symposium (International) on Combustion, pages 17011708

Laufer J (1953) The Structure of Turbulence in Fully Developed Pipe Flow. Technical Report Report 1174, National Bureau of Standards

Liu Y, Lenze B, Leuckel W (1989) Investigation on the Laminar and Turbulent Burning Velocities of Premixed Lean and Rich Flames of CH4-H2-Air Mixtures. In 12th ICDERS, Ann Arbor
Metghalchi M, Keck JC (1980) Laminar burning velocity of propane-air mixtures at high temperature and pressure. Combustion and Flame, 38:143-154

Metghalchi M, Keck JC (1982) Burning Velocities of Mixtures of Air with Methanol, Isooctane, and Indolene at High Pressure and Temperature. Combustion and Flame, 48:191-210

Noh WF, Woodward P (1976) SLIC (Simple Line Interface Calculation). In A.I. van de Vooren and P.J. Zandbergen, editors, Proceedings of the Fifth International Conference on Numerical Methods in Fluid Dynamics, volume 59 of Lecture Notes in Physics

Pineau JP, Giltaire M, Dangreaux J (1980) Propagation d'explosions de poussieres dans les canalisations. Technical Report Note 1230-98-80, Centre d'etudes et recherches des Charbonnages de France

Pineau JP, Ronchail G (1987) Propagation of Coal Dust Explosions in Pipes. In K.I.Cashdollar and M.Hertzberg, editors, Industrial Dust Explosions, ASTM STP 958, pages 74-89, Philadelphia. American Society for Testing and Materials

Ramaprian BR, Tu SW (1983) Fully developed periodic turbulent pipe flow. Part 2. The detailed strucutre of the flow. J. Fluid Mech., 137:59-81

Tonello NA (1995) An Experimental and Computational Study of the Mechanisms of Detonation Transmission in Layered $\mathrm{H}_{2}-\mathrm{O}_{2}$ Mixtures. Ph.D. thesis, The University of Michigan, Ann Arbor, Michigan

Trautwein SE (1989) Untersuchung des Einflusses der Turbulenz auf die Flammenausbreitung unter motorischen Bedingungen. Dissertation, RWTH Aachen

Trautwein SE, Grudno A, Adomeit A (1990) The Influence of Turbulence Intensity and Laminar Flame Speed on Turbulent Flame Propagation under Engine Like Conditions. 23rd Symposium (International) on Combustion, pages $723-728$

Tsuji Y, Morikawa Y (1982) LDV mesurements of an airsolid two-phase flow in a horizontal pipe. J. Fluid Mech., 120:385-409

Tsuji Y, Morikawa Y, and Shiomi H (1984) LDV mesurements of an air-solid two-phase flow in a vertical pipe. J. Fluid Mech., 139:417-434

Tu SW, Ramaprian BR (1983) Fully developed periodic turbulent pipe flow. Part 1. Main experimental results and comparison with predictions. J. Fluid Mech., 137:31-58

Vogl A (1992) Explosionsabläufe in Rohrleitungen - Untersuchungen im pneumatischen Fördersystem. In VDIBericht Nr. 701, pages 143-166

Vogl A (1995) Ablauf von Staubexplosionen in pneumatischen Saug-Flug-Förderanlagen. Angewandte Systemsicherheit und Arbeitsmedizin. Asanger Verlag, Heidelberg. Dissertation, RWTH Aachen

Vogl A (1996) Flame propagation in tubes of pneumatic conveying systems and exhaust equipment. In 29th Loss Prevention Symposium, New Orleans, 25.-29.2.96

Zhang F, Grönig H (1991) Transition to detonation in corn starch dust- oxygen and air mixtures. Combustion and Flame, 86:21-32 